\title{
3D and 4D Bioprinting of the Myocardium: Current Approaches, Challenges, and Future Prospects
}

\author{
Chin Siang Ong, ${ }^{1,2}$ Lucy Nam, ${ }^{1}$ Kingsfield Ong, ${ }^{3}$ Aravind Krishnan, ${ }^{1}$ Chen Yu Huang, \\ Takuma Fukunishi, ${ }^{1}$ and Narutoshi Hibino $\mathbb{1}^{1}$ \\ ${ }^{1}$ Division of Cardiac Surgery, Johns Hopkins Hospital, Baltimore, MD, USA \\ ${ }^{2}$ Division of Cardiology, Johns Hopkins Hospital, Baltimore, MD, USA \\ ${ }^{3}$ Department of Cardiac, Thoracic and Vascular Surgery, National University Heart Centre, Singapore \\ Correspondence should be addressed to Narutoshi Hibino; nhibino1@jhmi.edu
}

Received 24 November 2017; Revised 4 March 2018; Accepted 15 March 2018; Published 22 April 2018

Academic Editor: GeunHyung Kim

Copyright (c) 2018 Chin Siang Ong et al. This is an open access article distributed under the Creative Commons Attribution License, which permits unrestricted use, distribution, and reproduction in any medium, provided the original work is properly cited.

\begin{abstract}
3D and 4D bioprinting of the heart are exciting notions in the modern era. However, myocardial bioprinting has proven to be challenging. This review outlines the methods, materials, cell types, issues, challenges, and future prospects in myocardial bioprinting. Advances in 3D bioprinting technology have significantly improved the manufacturing process. While scaffolds have traditionally been utilized, 3D bioprinters, which do not require scaffolds, are increasingly being employed. Improved understanding of the cardiac cellular composition and multiple strategies to tackle the issues of vascularization and viability had led to progress in this field. In vivo studies utilizing small animal models have been promising. 4D bioprinting is a new concept that has potential to advance the field of 3D bioprinting further by incorporating the fourth dimension of time. Clinical translation will require multidisciplinary collaboration to tackle the pertinent issues facing this field.
\end{abstract}

\section{Introduction}

It has been reported that, in the United States, 1 in 7 deaths are attributable to coronary artery disease (CAD) and the estimated incidence of myocardial infarction is 790,000 per year [1]. Despite advances in medical therapy, fibrotic myocardial scar tissue formation after ischemia leading to depressed heart function is generally considered an irreversible process, short of a heart transplant. There have been many attempts at cardiac regeneration, but extensive data suggests transplanted cells, mostly delivered by injection, do not survive in the long term [2]. Cardiac tissue engineering [3] has thus been developed as a promising alternative in the treatment of ischemic heart disease, by cardiac regeneration. One of the newer methods of cardiac tissue engineering is three-dimensional (3D) bioprinting. 3D bioprinting has shown varying degrees of success, where optimization of the tissue engineering process has led to meaningful preclinical applications [4]. Meanwhile, the concept of adding time as an additional dimension to $3 \mathrm{D}$ bioprinting has led to the development of a new field of $4 \mathrm{D}$ bioprinting. This article aims to summarize the advances and challenges of $3 \mathrm{D}$ and $4 \mathrm{D}$ bioprinting of the myocardium.

\section{3D Bioprinting Methods}

3D printing, or additive manufacturing, refers to the creation of physical 3D objects by deposition of material in a successive layered approach, guided by previously specified custom digital designs, and has led to multiple innovations in cardiovascular medicine [5]. This technology has enabled the production of patient-specific 3D objects with varied geometries, sometimes complex, which have clinical applications in medical education, functional flow modelling, procedural planning, and device innovation. In addition, the development of solvent-free, aqueous-based systems have enabled the $3 \mathrm{D}$ printing of biomaterials into $3 \mathrm{D}$ scaffolds or molds that could be used for transplantation with or without seeded cells [6]. 


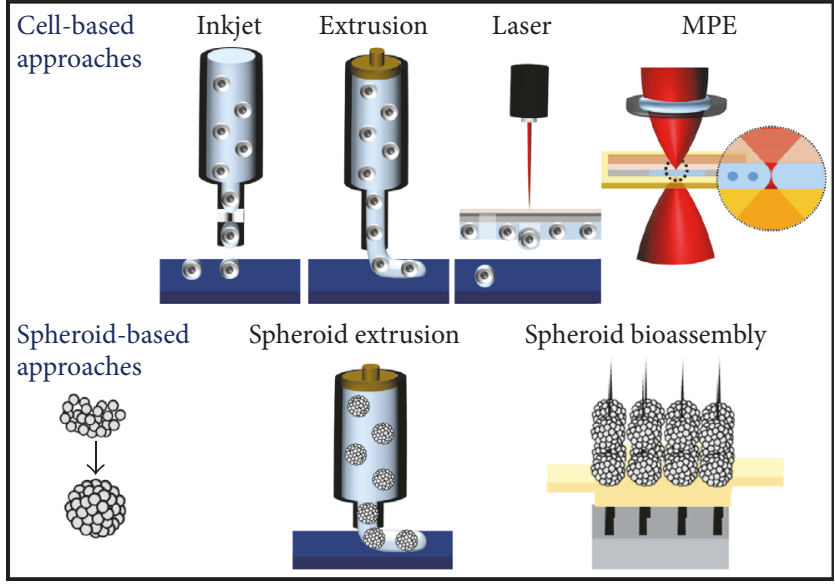

(a)

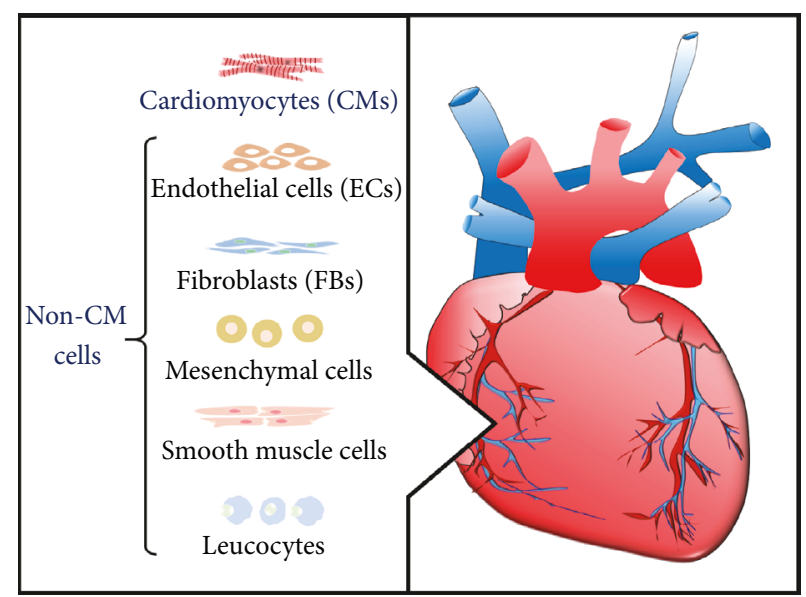

(b)

FIGURE 1: 3D bioprinting of the myocardium: methods (a) and cell types (b).

$3 \mathrm{D}$ bioprinting $[4,7]$ is a subset of $3 \mathrm{D}$ printing and involves the precise positioning of biomaterials and living cells in a layered approach, with spatial control over the arrangement of these functional components. Advances in $3 \mathrm{D}$ bioprinting have achieved substantial success in bioengineering of living tissues and organs. Methods of 3D bioprinting include inkjet bioprinting, microextrusion bioprinting, laser-assisted bioprinting, multiphoton excitation (MPE) based fabrication $[4,8]$, and spheroid-based approaches (Figure 1).

In inkjet bioprinting, thermal and piezoelectric drop-ondemand $3 \mathrm{D}$ bioprinters are most frequently utilized [4, 9, 10]. Thermal printing uses heat to create a vapour bubble in the biomaterial containing the cells of interest, which is then deposited via a nozzle. Piezoelectric 3D inkjet bioprinters work by applying different voltages to a piezoelectric crystal within the bioprinter. This generates the pressure required to eject the biomaterial containing the cells of interest out of the nozzle [4]. This is commonly used as the process is generally fast and inexpensive [9]. However, most of the problems arise due to clogging of the nozzle, which affects bioprinting precision and droplet direction [10]. Laser-assisted bioprinting or laser-induced forward transfer (LIFT) transfers the energy from a laser beam to a ribbon, which in turn deposits the cell-containing biomaterial onto a receiving substrate $[4,11,12]$. This mechanism overcomes the problems of nozzle clogging and permits high resolution bioprinting $[12,13]$. Microextrusion bioprinting works by robotically dispensing cell-containing biomaterials with the aid of mechanical (piston or screw) or pneumatic systems [14-16]. Although microextrusion bioprinting may appear primitive with low resolution, it has a significant advantage of being able to deposit high density biomaterial with high viability [15]. MPE-based bioprinting boasts high resolution by cross-linking polymers and proteins using photo energy $[8,17]$. In addition, newer techniques, combinations, and commercial variants have also emerged in recent years [18, 19]. Depending on the type of $3 \mathrm{D}$ bioprinting methods used, high resolution bioprinting can achieve resolutions less than $100 \mathrm{~nm}[20]$.

\section{3D Bioprinting Materials}

3.1. Scaffold-Dependent 3D Bioprinting. Scaffold-dependent [18] 3D bioprinting of myocardial tissue is more common and requires the incorporation of biomaterials, in the form of scaffolds or bioink [21-23]. The biomaterials utilized to 3D bioprint myocardial tissue include alginate, collagen, gelatin, hyaluronic acid, and decellularized extracellular matrix scaffolds (Table 1).

In the late 1990s, Eschenhagen and colleague successfully fabricated contractile myocardium made of chick embryonic cardiomyocytes, in a collagen matrix [31]. Since then most of the work has been centred on scaffold-dependent 3D bioprinting. However, there are limitations with the utilization of biomaterials, such as immunogenicity to the scaffold, degeneration of biomaterials, toxicity caused by the degraded products, and the additional costs in procuring the biomaterials [32].

3.1.1. Alginate. Alginate hydrogels have demonstrated high applicability as scaffolds for $3 \mathrm{D}$ printing [33]. Alginate is notable in its ability to make hydrogels at in vivo physiologic conditions, demonstrating therapeutic viability. In addition, alginate's ability to form a gel pore network that allows diffusion of nutrients and waste materials is vital for the functionality of bioprinted myocardium to function like native myocardium. Finally, given its extensive use in tissue engineering, alginate culturing methods are well characterized and reproducible for clinical evaluation.

Bioprinting of myocardium with a scaffold made from alginate was described by Gaetani et al. [24], who used the method of pressure-based extrusion to bioprint a patch of tissue that demonstrated cardiogenic potential. The group used human cardiac-derived cardiomyocyte progenitor cells 
TABLE 1: Examples of 3D bioprinting of the myocardium: methods, cell types, and scaffolds used.

\begin{tabular}{lccr}
\hline 3D bioprinting method & Cell type used & Scaffold & Reference \\
\hline Pressure based extrusion & hCMPCs & Alginate & Gaetani et al. [24] \\
\hline $\begin{array}{l}\text { Multihead tissue/organ building } \\
\text { system }\end{array}$ & Rat myoblast cells & $\begin{array}{c}\text { Decellularized } \\
\text { extracellular matrix }\end{array}$ & Pati et al. [25] \\
\hline Tissue printing & hCMPCs & Hyaluronic acid/gelatin & Gaetani et al. [16] \\
\hline Microcontact printing & $\begin{array}{c}\text { Double transgenic embryonic stem } \\
\text { cells (mouse embryonic fibroblast }+ \\
\text { embryonic stem cell) }\end{array}$ & Scaffold free & Atmanli and Domian [26] \\
\hline Spheroid extrusion & $\begin{array}{c}\text { Leghorn chicken atrioventricular } \\
\text { cells, human vascular endothelial } \\
\text { cells }\end{array}$ & Collagen & Jakab et al. [27, 28] \\
\hline Spheroid bioassembly & hiPSC-CMs & Scaffold free & Ong et al. [29, 30] \\
\hline
\end{tabular}

(hCMPCs) to construct the patch, which demonstrated viability after both one day and one week of culture. In addition, cells bioprinted in the cardiogenic patch demonstrated the ability to migrate from the alginate scaffold and form tubularlike structures, indicating the potential use of this method for therapeutic cell delivery, while retaining functional properties of cardiomyocytes.

3.1.2. Collagen. Jakab et al. [27] demonstrated the use of a "biopaper" hydrogel scaffold composed of rat-tail collagen type 1, on which spheroids of leghorn chicken atrioventricular cells (cAV cells) and human vascular endothelial cells (HUVECs) were printed. The bioprinted spheroid-biopaper tissue successfully fused and demonstrated synchronous beating at 90 hours similar to that of the native chicken cardiomyocytes. In addition, at 90 hours, there was evidence of early vascularization, with regions of elongated endothelial cells that formed vessel-conduit-like structures, signs of longterm viability of this tissue.

The biopaper hydrogel used in this printing method provided the appropriate environment for the spheroids printed on it to fuse and form functional tissue. Jakab et al. note that the biopaper was soft enough to allow the smooth deposition of the spheroids and also provided the appropriate environment for fusion and cell movement. These elements allowed the tissue to properly assemble into functional myocardium.

3.1.3. Hyaluronic Acid/Gelatin. Gaetani et al. [16] demonstrated the use of another scaffold, hyaluronic acid/gelatin, for tissue bioprinting with hCMPCs. Utilizing a murine model of myocardial infarction, the application of the bioprinted myocardium led to a significant reduction in adverse myocardial remodelling, which is thought to be implicated in the exacerbation of progressive heart failure [34]. Murine hearts that received the bioprinted tissue patch also demonstrated improved cardiac function and long-term engraftment of the hCMPCs.

Hyaluronic acid is a naturally occurring molecule in the ECM and thus has long been used as a scaffold for tissue engineering [35]. It is notable for its tensile strength as a biomaterial, which is important in beating myocardium to resist postengraftment destruction. The hyaluronic acid/gelatin complex is attractive due to its demonstrated safety in native tissue when implanted [36].

3.1.4. Decellularized Extracellular Matrix. Cell seeding onto decellularized extracellular matrix from cardiac tissue is an established technique to grow myocardium in vitro. Oberwallner et al. [37] demonstrated the ability to decellularize human myocardium to yield heart decellularized extracellular matrix (hdECM). Tissue retrieved from patients undergoing implantation of a ventricular assist device was decellularized using a detergent method and then incubated in fetal bovine serum, yielding hdECM with native-like levels of collagen, laminin, and fibronectin. Human mesenchymal stem cells, murine cardiomyocytes derived from induced pluripotent stem cells, and naive neonatal mouse cardiomyocytes were seeded on the human hdECM and demonstrated greater proliferation and viability compared to standard culture. Cells seeded on to the human hdECM beat synchronously. However, the use of hdECM is just beginning to flourish.

Pati et al. [25, 38] demonstrated the use of decellularized extracellular matrix as a bioink for 3D bioprinting. In their experiment, porcine heart extracellular matrix was decellularized by a series of biochemical processes to yield hdECM bioink. The resulting hdECM had near-native levels of collagen and glycosaminoglycans, providing a viable environment for 3D bioprinting. A printing method referred to as the multihead tissue/organ building system [39] was used to simultaneously print the hdECM bioink with rat myoblasts to produce a tissue block that demonstrated viability at 24 hours. Gene and protein expression of the myoblasts printed with the bioink demonstrated vital components for graft survival and function. The microarchitecture of the graft also demonstrated native-like organization, suggesting the potential for these grafts to be used for myocardial reconstruction [25].

Jang et al. [40] have since demonstrated a novel technique of producing hdECM complexed with ultraviolet radiationtreated vitamin B2 (VB2). The resulting bioink, printed with human cardiac progenitor cells, was characterized by stiffness similar to native myocardium. The improved tissue stiffness 
is thought to be the result of VB2, which forms stable crosslinks when exposed to ultraviolet radiation. Furthermore, the cardiac progenitor cells actively proliferated and showed signs of effective differentiation.

The extracellular matrix microenvironment is thought to be critical in promoting and guiding stem cell differentiation $[38,40,41]$. The various proteins within the hdECM allowed for the cells bioprinted with this bioink to differentiate and organize with native-like instructions. Furthermore, the bioink provided a structure that supported the bioprinted grafts' long-term viability. Furthermore, the use of fortifying materials such as VB2 may represent approaches to better supporting bioprinted cells on hdECM that closely resembles native ECM [40].

3.2. Scaffold-Free 3D Bioprinting. Despite the successes of the experiments detailed so far, the use of scaffolds is not without issues. Scaffolds face the issue of rapid degeneration leading to limited mechanical/physical stability [26, 42]. Investigations into scaffold-free 3D bioprinting of myocardium have yielded some positive results.

Atmanli and Domian [26] demonstrated the novel use of microcontact printing to bioprint functional cardiac tissue that preserves the unique native architecture of myocardium. They did so using double transgenic murine committed ventricular progenitors (CVPs). CVPs resemble cardiac myocytes on a number of parameters including expression of myocardial markers, as well as exhibiting functional and structural properties [43]. Normally scaffolds provide extracellular cues for cardiac progenitor cells to organize into myocytes. In Atmanli and Domian's microcontact printing method, they addressed the need of a scaffold by relying on microtextured polydimethylsiloxane (PDMS) stamps instead, to guide the microarchitecture of the bioprinted tissue. The resulting tissue represented the characteristic anisotropy of native myocardium, which is critical for its mechanical and electrophysiological properties [44-46]. This experiment did not test the in vivo therapeutic capacity of myocardium bioprinted in this manner but nonetheless represents a pivotal early step in scaffold-free 3D bioprinting of myocardial tissue.

Tissue-spheroid-based organ bioprinting is emerging as a major alternative to traditional scaffold-based tissue bioprinting [47]. Tissue spheroids are three-dimensional, organized clusters of cells in bulk. When tissue spheroids are placed near each other, surface tension causes them to fuse into a "living material." This material demonstrates the physiological properties of the native tissue the spheroid cells were derived from. Spheroids provide several advantages in their ability to be organized at a cellular level like organoids in a native tissue, as well as the ability to seamlessly assemble spheroids into larger structures that still preserve the characteristics of the native tissue without the constraints of scaffolds [18]. Tissue spheroids composed of rat neonatal ventricular cardiomyocytes, human dermal fibroblasts, and human coronary microartery endothelial cells were used to form a contractile cardiac patch that remained viable after being transplanted into rats by Noguchi et al. [48]. This demonstrates the potential for myocardium to be constructed using tissue spheroids.
Recent technological advancements have led to $3 \mathrm{D}$ bioprinters capable of assembling spheroids, with precise positioning of individual spheroids [18]. This method uses a robotic arm to pick up preassembled tissue spheroids using vacuum suction and impale them onto microneedles arranged in an array. The spheroids subsequently fuse and functional tissues are then removed from the needle arrays for further maturation. This technique offers a reproducible, reliable, and precise technique to bioprint tissue spheroids into organized tissue.

Ong et al. [29, 30] used this method to bioprint tissue spheroids composed of human induced pluripotent stem cell-derived cardiomyocytes (hiPSC-CMs), fibroblasts, and endothelial cells into myocardial patches. The cardiac patches demonstrated spontaneous beating, as well as ventricularlike action potential waveforms and uniform electrical conduction throughout the patches. These patches were then implanted in vivo into a rodent model and demonstrated engraftment and vascularization, suggesting the therapeutic regenerative potential of this scaffold-free $3 \mathrm{D}$ bioprinting method.

\section{Cell Types}

Cells used in these experiments can be broadly classified into nonhuman and human cells. Some methods used cell mixtures from both types as well. Rat myoblast cells (L6 cell line) have been shown to differentiate into functioning cardiac tissue in a variety of experiments [25, 49]. In Jakab et al.'s [27] study, embryonic myocardium was harvested from leghorn chickens and cardiomyocytes were bioprinted on a biopaper hydrogel. Murine committed ventricular progenitors were used in Atmanli and Domian's scaffold-free method, due to the cells' similarity to human cardiomyocytes by a number of parameters $[26,43]$.

In terms of human cells [50], hCMPCs and hiPSC-CMs are popular choices for 3D bioprinting [16, 17, 24, 26, 29, 30]. These cells exhibit genetic profiles and express proteins that are vital for differentiation into functional myocardium, and they demonstrate the characteristics of native myocardium when bioprinted in the methods described above.

Mature cardiomyocytes are the result of differentiation of human cardiomyocyte progenitor cells produced from human induced pluripotent stem cells or human embryonic stem cells [51]. Various protocols have been established to differentiate human pluripotent stem cells into beating cardiomyocytes [52, 53], and various groups have demonstrated the therapeutic potential in the repair of damaged myocardium and cardiac regeneration [54-56].

However, immaturity of stem cell-derived cardiomyocytes, due to incomplete maturation [57], remains a major obstacle and promoting cardiomyocyte maturation is important in order to achieve the final goal of cardiac regeneration [58]. Chong et al. [59] observed in a nonhuman primate model of myocardial ischemia-reperfusion that treatment with human embryonic stem cell-derived cardiomyocytes (hESC-CMs) led to significant remuscularization, albeit with nonfatal ventricular arrhythmias, due to incomplete maturation of hESC-CMs. In addition, other issues regarding 
tumorigenesis, graft viability, and immunological rejection [60] have to be addressed as well, before human studies can be conducted.

$3 \mathrm{D}$ printing scaffolds separately and then seeding cells have been a major area of research in myocardial tissue engineering as well, though there are significant challenges associated with this method including its resource- and timeintensive requirements [25]. Certain novel methods in 3D printed scaffolds with subsequent cell seeding have yielded viable myocardium $[12,17,19]$ with the use of C2C12 myoblast cells, human mesenchymal stem cells (hMSCs), and hiPSCCMs as the seeded cells. Reviewing cell types that have been seeded into 3D printed scaffolds and the results of these experiments can give new ideas regarding potential cell types for 3D bioprinting.

\section{Cardiac Cellular Composition}

A clear understanding of the myocardial cellular composition is crucial in bioengineering of the myocardium. The specific cellular composition of cardiomyocytes and noncardiomyocytes in the native human myocardium has been a subject of debate [61, 62]. Previously, studies found that fibroblasts represented the majority of noncardiomyocytes but modern techniques in recent literature have showed this may not be true [63-65]. Some of the reasons to explain this discrepancy include utilization of nonspecific markers and reagents, estimation used in stereological approaches, failure to completely dissociate cells, and low cell viability during flow cytometry. These factors can significantly alter the overall distribution. Most recently, Pinto et al. argued that the majority of noncardiomyocytes are endothelial cells [65]. Using improved cell isolation techniques and flow cytometry, they found that more than $60 \%$ of the noncardiomyocyte cell population consisting of endothelial cells and fibroblasts contributed to less than $20 \%$, while the remaining cell types included leucocytes, pericytes, and other resident mesenchymal cells. Subsequently, analysis of the human heart also demonstrated predominance of endothelial cells, corresponding to histological studies of the cardiac myocardium conducted much earlier, decades ago [66-68].

Variations in the cell composition implemented during the fabrication of non-3D printed engineered heart tissue (EHT) influenced the structure and function of the cardiac patch [69-72]. Functionally, forces generated by the EHT made from pure cardiomyocytes were three time less than those made from a mix of native cardiomyocytes and noncardiomyocytes [72]. Structurally, the addition of fibroblasts to cardiomyocytes improved cellular architecture with a more uniformed layout [71]. Increased microvascularization was observed with the addition of other cells, such as endothelial cells, fibroblasts, stromal cells, and mesenchymal cells $[69,70,73]$. EHT supplemented with noncardiomyocytes performed better than EHT made of pure cardiomyocytes when implanted in vivo $[69,70]$. Tulloch and colleagues demonstrated perfusion by host erythrocytes as early as 1 week in implanted EHTs which were supplemented with human umbilical vein endothelial cells, human marrow stromal cells, and mouse embryonic fibroblasts [69].
Likewise, encouraging results were reported in the $3 \mathrm{D}$ bioprinting of the myocardium when a mixture of cardiomyocytes and noncardiomyocytes were utilized [12, 17]. Gaebel and colleagues [12] seeded a cardiac tissue patch with HUVECs and hMSCs in a systemic pattern. This was based on evidence that suggested hMSCs could ameliorate angiogenesis in postinfarcted myocardium, affecting cell repair and regeneration processes. They were perceived to be able to prevent apoptosis induced by hypoxic endothelial cells, which in turn promoted angiogenesis and cell survival [74]. Gao and colleagues [17] seeded their native-like ECM scaffold with cardiomyocytes, endothelial cells, and smooth muscle cells derived from human cardiac lineage induced pluripotent cells with good effect. Synchronous contractility was observed on their cardiac patch 1 day after seeding and by day 7 demonstrated good electrophysical properties of cardiac muscle function and the presence of the genetic expressions necessary for contractility.

\section{Viability and Vascularization}

For $3 \mathrm{D}$ bioprinting of the myocardium to be feasible in clinical application, tissue perfusion and vascularization are vital. Adequate blood supply to the transplanted $3 \mathrm{D}$ bioprinted myocardial graft is important for the long-term durability and viability of the graft, after in vivo implantation. For meaningful clinical applications, the myocardial tissues constructed have to be of a reasonable size, and due to this, diffusion alone is usually insufficient to maintain the delivery of oxygen and nutrients necessary for cell survival. To achieve optimal oxygen and nutrient delivery in vivo, living cells have to be within 100-200 $\mu \mathrm{m}$ from its supplying capillaries [75].

6.1. Vascularization Strategies. There are a number of vascularization strategies that have been studied with varying degree of success, which could be relevant to engineer an organized vasculature within the $3 \mathrm{D}$ bioprinted myocardium in a hybrid fashion. Vascularization via cell to cell interaction relies on utilizing proangiogenic cells that are cocultured to enhance vascularization [76]. The addition of endothelial cells in vitro during cell culture have demonstrated improved growth and stabilization of vascular networks in engineered tissues, such as skin and skeletal muscle when implanted in vivo $[76,77]$. Other studies have shown useful application of angiogenic factors, such as vascular endothelial growth factor (VEGF), stromal cell-derived factor 1 (SDF1), or angiopoietin-1 to hasten and promote the formation of vessel networks [77-79]. In essence, endothelial cells are cultured on polyethylene glycol hydrogels with covalently bound angiogenic factors. The bioactive ligands in the hydrogel release the angiogenic factors to direct vessel formation [80]. Capillary like networks were observed as early as two days [81]. Schesny and colleague demonstrated preserved rodent heart function postinfarction with controlled release of SDF1 [79]. Improved regeneration and cell survival in ischemic murine hindlimbs of murine model were reported with the utilization of VEGF releasing hydrogels seeded with endothelial cells [82]. SDF1 has also been shown to enhance neovascularization in ovine hearts postmyocardial infarction 
[83]. However, these techniques allow limited control over the exact architectural alignment and specifications of the resultant vascular networks formed.

In order to fabricate more precise vascular networks that resemble a native vascular tree, vascularization with the aid of sacrificial materials had been investigated and had shown promise [84-86]. Miller and colleague were able to produce a $3 \mathrm{D}$ printed vascular network lined with endothelial cells, using carbohydrate filaments as sacrificial material, which were robust enough to be perfused with pulsatile and high-pressure flows [87]. Another construct using micropores proved successful in sustaining perfusion and thereby maintaining the metabolic processes in a threedimensionally engineered hepatocyte tissue [85]. Similarly, spatial micropatterning is another useful technique for the fabrication of an organized micro- and nanovasculature. Culver and colleagues were able to use photon lasers to create three-dimensional pathways in a hydrogel lined with angiogenic factors, guiding the growth of endothelial cells into an intricate vascular network [88]. This laser guided cell patterning for the formation of tubular networks within collagen hydrogels was also demonstrated by Hribar and colleagues [89]. Ultrasound standing wave fields are a noninvasive spatial micropatterning technique that directs endothelial cells to form vascular networks within engineered tissues, by controlling acoustics in a sound field [90-93]. Overall, these methods provide hope for further research to better engineer viable vessel networks, enabling prompt and adequate perfusion of implanted tissues in vivo.

6.2. Vascularizing 3D Bioprinted Myocardium. Most the work with regard to myocardial vascularization has been initially conducted using non-3D printed EHT in animal models $[69,94,95]$. Rapid angiogenesis of implanted EHT has been previously demonstrated, with a dense network of microvascularization shown within 2 weeks of EHT implantation $[69,95]$ and host erythrocytes identified in these vessels [70]. Utilizing immunostaining with human-specific CD31 and $\alpha$ smooth muscle actin, Lesman and colleagues demonstrated the origins of both human (donor) and rat (host) vasculature in the human EHT grafts that were implanted in murine hearts [70].

The construction of thick viable myocardial tissue is another challenge. Shimizu and colleagues [96] initially tried to implant cell sheets thicker than 3 layers in one sitting, but due to a lack of intrinsic microvasculature, the implanted tissue quickly became necrotic. Shimizu et al. subsequently managed to construct thick viable myocardium by multiple implantations of thin cell sheets at intervals of 1 to 2 days. The macrovasculature is also important to perfuse thick myocardial tissue grafts. Several innovative designs to address this issue have shown promising results [97-100]. Essentially, the concept involves culturing EHT with arterial and venous vessels in a medium, to promote angiogenesis between the micro- and macrovasculatures. The addition of vascular endothelial cells has also been shown to promote vascularization in $3 \mathrm{D}$ bioprinted cardiac constructs, such as in experiments by Jakab et al. [27, 28].
3D bioprinted myocardium has demonstrated vascularization and significant therapeutic effects, when implanted into animal hearts. Gaebel and colleagues demonstrated the presence of primitive vascular networks in 3D bioprinted myocardium constructed by LIFT 3D bioprinting that were seeded with human mesenchymal cells and HUVECs [12]. Eight weeks after infarction, fluorescent-labelled tracings revealed primitive vascular structures, high density of capillary networks, integration with host vasculature, and significant improvement in cardiac function. Similarly, Gaetani and colleagues also reported improvements in cardiac function, after applying 3D bioprinted hyaluronic acid/gelatin patches containing cardiac progenitor cells in postmyocardial infarction murine hearts [16]. These patches showed continued proliferation and maturation when analyzed at 4 weeks, suggesting durability and viability of 3D bioprinted cardiac patches [16]. Gao and colleagues observed increased population of arterioles in the cardiac patches constructed by MPE-based 3D bioprinting after implantation into murine hearts [17]. Contractility was observed from day 1 . At 4 weeks, significant therapeutic effects including reduction of infarct size and improved ejection fraction were noted. Our group also constructed scaffold-free 3D bioprinted myocardium and demonstrated viability, vascularization, and engraftment 1 week after implantation [29,30]. To surmise, multiple in vivo studies have demonstrated vascularization of engineered cardiac tissue and the addition of vascular cells in vitro may prove beneficial in 3D bioprinting of the myocardium in promoting viability, paving the way for possible clinical application in the future.

\section{4D Bioprinting}

4D bioprinting incorporates the fourth dimension of "time" into 3D bioprinting. This includes instances in which objects can change their shape based on the presence of external stimuli, such as when cell fusion or self-assembly occurs [101]. With the aim of creating in vitro models that resemble tissue structures found in nature, $4 \mathrm{D}$ bioprinting promotes dynamic, structural, and cellular changes of a tissue over time, overcoming the static nature of 3D bioprinting [102].

As a new field, the classification and definition of $4 \mathrm{D}$ bioprinting are still not universally agreed upon. One classification proposed by An et al. defines $4 \mathrm{D}$ bioprinting to include several approaches; two of these approaches include the folding of tissue on a substrate into a desired shape and the self-assembly or self-organization of a tissue. To constitute $4 \mathrm{D}$ bioprinting, these transformations must be through the induction of a stimulus, rather than a change caused by natural processes [103]. Although the field of $4 \mathrm{D}$ bioprinting of the myocardium is still nascent, there is published literature that provides insights.

The first approach to $4 \mathrm{D}$ bioprinting prints tissue onto a substrate material (like a responsive hydrogel) and folds the tissue into a desired shape upon stimulus induction [103]. A number of researchers have worked on creating these shape-morphing substrates and scaffolds: Kirillova et al. experimented with printing shape-morphing hydrogel made of two different biopolymers (alginate and hyaluronic 
acid) to fabricate hollow self-folding tubes. These formed diameters comparable to those of the smallest blood vessels and maintained the viability of the printed cells for 7 days without a decrease in cell viability [104]. Apsite et al. created electrospun, porous multilayer scaffolds based on thermoresponsive polymers. At different temperatures in an aqueous environment, these scaffolds spontaneously rolled to form a tube with distinct layers. By adding collagen, cell viability and adhesion were improved [105]. Furthermore, self-healing hydrogels have been studied for therapeutic and biomedical applications. These hydrogels increase the lifetime of a functional material and also exhibit similar characteristics to human tissue [106]. Cardiomyocytes cultured in a $3 \mathrm{D}$ fibrin hydrogel environment that mimics the cell's natural environment were noted to remodel the construct to generate contractile force under electrical pacing conditions [107]. Culturing cardiomyocytes in a shape-morphing hydrogel can potentially aid the formation of more native-like 3D structures over time. With advances in bioprinting stimuliresponsive hydrogels and cardiomyocyte viability in hydrogels, further advances in this approach of $4 \mathrm{D}$ bioprinting can promote the ability to control the spatial arrangement of cells and internal structure of engineered myocardium.

The second approach is to promote the self-assembly of tissues through the stimulation of a printed structure. For example, printed microdroplets can precisely deposit cells in a particular pattern that will conform to another pattern upon stimulation [103]. There has been research that addressed the stimulation of cardiac tissue structures: Kaji et al. [108] analyzed the response to chemical stimuli on a single-cell level. With chemical stimuli, the myocytes conjugated to form gap junctions. A stimulus, caffeine, activates the gap junction communication whereas 1-octanol reversibly inhibits it. Our group has also used defined chemical factors to stimulate scaffold-free 3D bioprinted cardiac patches, to promote better tissue organization and maturity. Serpooshan et al. utilized Faraday waves as a stimulus to enable the aggregation of hiPSC-CMs into predefined 3D constructs [109]. With the ability to 3D bioprint cardiac tissue and a variety of stimulus mechanisms to induce tissue structural and cellular changes over time, 4D bioprinting of the myocardium remains an active area of research, and the considerable possibilities and potentially therapeutic benefits mean that novel work in this field will be highly impactful.

\section{Challenges and Future Prospects}

The choice of 3D bioprinting method and the decision whether to use scaffolds (and if so, which scaffold) are important considerations. During the delivery of the scaffold, cells are sometimes subjected to the unavoidable effects of thermal or vibrational energy and sheering forces, depending on the method of $3 \mathrm{D}$ bioprinting. Immunogenicity of the scaffolds is still an issue that has to be addressed. Further studies are needed to evaluate both the short and long-term effects of the by-products released during the breakdown of the scaffolds. Also, most scaffolds generated do not reflect the native architecture of adult myocardium. Decellularization of heart tissue can be a solution as it can create constructs that closely mimic native adult myocardium [110]. On the other hand, most scaffolds are notably porous in nature. This allows waste exchange and vascularization, which aid in graft survival in vivo. In addition, this porosity allows cell attachment and facilitates proliferation and maturation. Scaffolds also boast good tensile strength and the ability for the $3 \mathrm{D}$ bioprinting myocardium to withstand high pressure and the forces of myocardial contractions. 3D bioprinting using tissue spheroids is an attractive alternative as it allows customized tissue design without the use of scaffolds and avoids the problems with cell injury during the dispensing of the biomaterial. The production of a desired composition tissue spheroid is relatively straight forward, and it has shown to form cell-cell junctions spontaneously, demonstrating synchronous contraction and the development of primitive vascular like structures $[27,111,112]$. Overall, scaffold-free and scaffold-dependent options have their own advantages and drawbacks, with both showing evidence of continued progress and optimization.

Achieving the ideal cellular composition for cardiac constructs is still challenging, as we are still learning about the native human myocardium. This is complicated by the observations that altering the composition of noncardiomyocytes, such as fibroblasts and endothelial cells, affects the function, vascularization, and viability of the $3 \mathrm{D}$ bioprinted myocardium. Ultimately, there may not be a need for complete biomimicry for cardiac regeneration in terms of cell ratio of the cardiac construct, and further studies are needed to investigate this.

Regarding vascularization, significant progress has been reported. Early results from studies incorporating vascular cells into these models are encouraging, with therapeutic efficacy demonstrated in murine hearts. To construct a sizable, macroscale [113], mechanically robust and clinically meaningful 3D bioprinted myocardium, an established intrinsic vasculature for adequate perfusion is necessary to keep up with the high metabolic demands of contractility. Vascularization and scalability represent some of the greatest challenges for the progress of this technology. In addition, further testing in large animal models to evaluate the safety and therapeutic effects of these tissues is needed to provide preclinical data for possible future clinical applications. These results will significantly influence the future of this technology.

We have also listed potential avenues for time-varying $4 \mathrm{D}$ bioprinting to transform cardiac tissue engineering. $4 \mathrm{D}$ bioprinting utilizes stimuli-responsive materials and shapememory polymers to create dynamic 3D patterned biological structures that transform in shape and structure, in the presence of a stimulus [104]. Due to the early nature of 4D bioprinting of tissues, it is feasible that new approaches and technologies will emerge once more research is conducted. One possible limitation of 4D bioprinting is the presence of a stimulus, which may present the opportunity to damage and kill living cells. The stimulus must thus be tuned or titrated to ensure that this does not happen to a significant degree.

Overall, 4D bioprinting of the myocardium is in its infancy and although we are years, if not decades, away 
from major clinical application breakthroughs, the technology presents a number of important opportunities. First, by adding the time dimension to $3 \mathrm{D}$ bioprinted materials, $4 \mathrm{D}$ bioprinting offers the opportunity to build dynamic structures that better represent the types of tissue structures in vivo, for tissue regeneration. Furthermore, by designing structures that can respond to stimuli, we can build cardiac tissue models that respond to unique cell conditions and open their applicability to a variety of treatments.

In conclusion, the evidence presented in this review gives hope for the future. Continued progress will require close collaboration among physicians, scientists, biomedical engineers, and experts from other fields, to optimize this approach combining traditional cardiac tissue engineering techniques with 3D and 4D bioprinting.

\section{Conflicts of Interest}

The authors declare that they have no conflicts of interest.

\section{References}

[1] E. J. Benjamin, M. J. Blaha, S. E. Chiuve et al., "Heart Disease and Stroke Statistics-2017 Update: A Report From the American Heart Association," Circulation, 2017.

[2] J. C. Garbern and R. T. Lee, "Cardiac stem cell therapy and the promise of heart regeneration," Cell Stem Cell, vol. 12, no. 6, pp. 689-698, 2013.

[3] M. N. Hirt, A. Hansen, and T. Eschenhagen, "Cardiac tissue engineering: state of the art," Circulation Research, vol. 114, no. 2, pp. 354-367, 2014.

[4] S. V. Murphy and A. Atala, " 3D bioprinting of tissues and organs," Nature Biotechnology, vol. 32, no. 8, pp. 773-785, 2014.

[5] M. Vukicevic, B. Mosadegh, J. K. Min, and S. H. Little, "Cardiac 3D Printing and its Future Directions," JACC: Cardiovascular Imaging, vol. 10, no. 2, pp. 171-184, 2017.

[6] A. V. Borovjagin, B. M. Ogle, J. L. Berry, and J. Zhang, "From Microscale Devices to 3D Printing: Advances in Fabrication of 3D Cardiovascular Tissues," Circulation Research, vol. 120, no. 1, pp. 150-165, 2017.

[7] A. S. T. Smith, J. Macadangdang, W. Leung, M. A. Laflamme, and D.-H. Kim, "Human iPSC-derived cardiomyocytes and tissue engineering strategies for disease modeling and drug screening," Biotechnology Advances, vol. 35, no. 1, pp. 77-94, 2017.

[8] P. J. Campagnola, D. M. Delguidice, G. A. Epling et al., "3-dimensional submicron polymerization of acrylamide by multiphoton excitation of xanthene dyes," Macromolecules, vol. 33, no. 5, pp. 1511-1513, 2000.

[9] T. Xu, C. A. Gregory, P. Molnar et al., "Viability and electrophysiology of neural cell structures generated by the inkjet printing method," Biomaterials, vol. 27, no. 19, pp. 3580-3588, 2006.

[10] T. Xu, H. Kincaid, A. Atala, and J. J. Yoo, "High-throughput production of single-cell microparticles using an inkjet printing technology," Journal of Manufacturing Science and Engineering, vol. 130, no. 2, pp. 0210171-0210175, 2008.

[11] J. A. Barron, B. R. Ringeisen, H. Kim, B. J. Spargo, and D. B. Chrisey, "Application of laser printing to mammalian cells," Thin Solid Films, vol. 453-454, pp. 383-387, 2004.
[12] R. Gaebel, N. Ma, J. Liu et al., "Patterning human stem cells and endothelial cells with laser printing for cardiac regeneration," Biomaterials, vol. 32, no. 35, pp. 9218-9230, 2011.

[13] M. Ali, E. Pages, A. Ducom, A. Fontaine, and F. Guillemot, "Controlling laser-induced jet formation for bioprinting mesenchymal stem cells with high viability and high resolution," Biofabrication, vol. 6, no. 4, Article ID 045001, 2014.

[14] D. M. Kirchmajer, R. Gorkin III, and M. In Het Panhuis, "An overview of the suitability of hydrogel-forming polymers for extrusion-based 3D-printing," Journal of Materials Chemistry B, vol. 3, no. 20, pp. 4105-4117, 2015.

[15] I. T. Ozbolat and M. Hospodiuk, "Current advances and future perspectives in extrusion-based bioprinting," Biomaterials, vol. 76, pp. 321-343, 2016.

[16] R. Gaetani, D. A. M. Feyen, V. Verhage et al., "Epicardial application of cardiac progenitor cells in a 3D-printed gelatin/hyaluronic acid patch preserves cardiac function after myocardial infarction," Biomaterials, vol. 61, pp. 339-348, 2015.

[17] L. Gao, M. E. Kupfer, J. P. Jung et al., "Myocardial Tissue Engineering with Cells Derived from Human-Induced Pluripotent Stem Cells and a Native-Like, High-Resolution, 3Dimensionally Printed Scaffold," Circulation Research, vol. 120, no. 8, pp. 1318-1325, 2017.

[18] N. I. Moldovan, N. Hibino, and K. Nakayama, "Principles of the kenzan method for robotic cell spheroid-based threedimensional bioprinting," Tissue Engineering - Part B: Reviews, vol. 23, no. 3, pp. 237-244, 2017.

[19] W. Y. Yeong, N. Sudarmadji, H. Y. Yu et al., "Porous polycaprolactone scaffold for cardiac tissue engineering fabricated by selective laser sintering," Acta Biomaterialia, vol. 6, no. 6, pp. 2028-2034, 2010.

[20] S. Tasoglu and U. Demirci, "Bioprinting for stem cell research," Trends in Biotechnology, vol. 31, no. 1, pp. 10-19, 2013.

[21] X. Cui, T. Boland, D. D. D’Lima, and M. K. Lotz, “Thermal inkjet printing in tissue engineering and regenerative medicine," Recent Patents on Drug Delivery and Formulation, vol. 6, no. 2, pp. 149-155, 2012.

[22] C. M. Murphy, M. G. Haugh, and F. J. O'Brien, “The effect of mean pore size on cell attachment, proliferation and migration in collagen-glycosaminoglycan scaffolds for bone tissue engineering," Biomaterials, vol. 31, no. 3, pp. 461-466, 2010.

[23] B. Mosadegh, G. Xiong, S. Dunham, and J. K. Min, "Current progress in 3D printing for cardiovascular tissue engineering," Biomedical Materials, vol. 10, no. 3, Article ID 034002, 2015.

[24] R. Gaetani, P. A. Doevendans, C. H. G. Metz et al., "Cardiac tissue engineering using tissue printing technology and human cardiac progenitor cells," Biomaterials, vol. 33, no. 6, pp. 17821790, 2012.

[25] F. Pati, J. Jang, D.-H. Ha et al., "Printing three-dimensional tissue analogues with decellularized extracellular matrix bioink," Nature Communications, vol. 5, article 3935, 2014.

[26] A. Atmanli and I. J. Domian, "Generation of aligned functional myocardial tissue through microcontact printing," Journal of visualized experiments, vol. 73, e50288 pages, 2013.

[27] K. Jakab, C. Norotte, B. Damon et al., "Tissue engineering by self-assembly of cells printed into topologically defined structures," Tissue Engineering - Part A., vol. 14, no. 3, pp. 413421, 2008.

[28] K. Jakab, C. Norotte, F. Marga, K. Murphy, G. VunjakNovakovic, and G. Forgacs, "Tissue engineering by selfassembly and bio-printing of living cells," Biofabrication, vol. 2, no. 2, Article ID 022001, 2010. 
[29] C. S. Ong, T. Fukunishi, A. Nashed et al., "Creation of cardiac tissue exhibiting mechanical integration of spheroids using 3D bioprinting," Journal of Visualized Experiments, vol. 2017, no. 125, Article ID e55438, 2017.

[30] C. S. Ong, T. Fukunishi, H. Zhang et al., "BiomaterialFree Three-Dimensional Bioprinting of Cardiac Tissue using Human Induced Pluripotent Stem Cell Derived Cardiomyocytes," Scientific Reports, vol. 7, no. 1, article no. 4566, 2017.

[31] T. Eschenhagen, C. Fink, U. Remmers et al., "Threedimensional reconstitution of embryonic cardiomyocytes in a collagen matrix: A new heart muscle model system," The FASEB Journal, vol. 11, no. 8, pp. 683-694, 1997.

[32] C. Norotte, F. S. Marga, L. E. Niklason, and G. Forgacs, "Scaffold-free vascular tissue engineering using bioprinting," Biomaterials, vol. 30, no. 30, pp. 5910-5917, 2009.

[33] T. Andersen, P. Auk-Emblem, and M. Dornish, "3D cell culture in alginate hydrogels," Microarrays, vol. 4, no. 2, pp. 133-161, 2015.

[34] J. Yang, Y. Liu, X. Fan, Z. Li, and Y. Cheng, "A pathway and network review on beta-adrenoceptor signaling and beta blockers in cardiac remodeling," Heart Failure Reviews, vol. 19, no. 6, pp. 799-814, 2014.

[35] J. S. Mao, H. F. Liu, Y. J. Yin, and K. De Yao, "The properties of chitosan-gelatin membranes and scaffolds modified with hyaluronic acid by different methods," Biomaterials, vol. 24, no. 9, pp. 1621-1629, 2003.

[36] A. C. Gaffey, M. H. Chen, C. M. Venkataraman et al., "Injectable shear-thinning hydrogels used to deliver endothelial progenitor cells, enhance cell engraftment, and improve ischemic myocardium," The Journal of Thoracic and Cardiovascular Surgery, vol. 150, no. 5, pp. 1268-1276, 2015.

[37] B. Oberwallner, A. Brodarac, Y.-H. Choi et al., "Preparation of cardiac extracellular matrix scaffolds by decellularization of human myocardium," Journal of Biomedical Materials Research Part A, vol. 102, no. 9, pp. 3263-3272, 2014.

[38] F. Pati and D.-W. Cho, "Bioprinting of 3D tissue models using decellularized extracellular matrix bioink," Methods in Molecular Biology, vol. 1612, pp. 381-390, 2017.

[39] J.-H. Shim, J.-S. Lee, J. Y. Kim, and D.-W. Cho, "Bioprinting of a mechanically enhanced three-dimensional dual cell-laden construct for osteochondral tissue engineering using a multihead tissue/organ building system," Journal of Micromechanics and Microengineering, vol. 22, no. 8, Article ID 085014, 2012.

[40] J. Jang, T. G. Kim, B. S. Kim, S.-W. Kim, S.-M. Kwon, and D.-W. Cho, "Tailoring mechanical properties of decellularized extracellular matrix bioink by vitamin B2-induced photocrosslinking," Acta Biomaterialia, vol. 33, pp. 88-95, 2016.

[41] F. Guilak, D. M. Cohen, B. T. Estes, J. M. Gimble, W. Liedtke, and C. S. Chen, "Control of stem cell fate by physical interactions with the extracellular matrix," Cell Stem Cell, vol. 5, no. 1, pp. 17-26, 2009.

[42] B. P. Chan and K. W. Leong, "Scaffolding in tissue engineering: general approaches and tissue-specific considerations," European Spine Journal, vol. 17, no. 4, pp. S467-S479, 2008.

[43] I. J. Dornian, M. Chiravuri, V. D. P. Meer et al., "Generation of functional ventricular heart muscle from mouse ventricular progenitor cells," Science, vol. 326, no. 5951, pp. 426-429, 2009.

[44] A. W. Feinberg, P. W. Alford, H. Jin et al., "Controlling the contractile strength of engineered cardiac muscle by hierarchal tissue architecture," Biomaterials, vol. 33, no. 23, pp. 5732-5741, 2012.
[45] L. D. Black, J. D. Meyers, J. S. Weinbaum, Y. A. Shvelidze, and R. T. Tranquillo, "Cell-Induced Alignment Augments Twitch Force in Fibrin Gel-Based Engineered Myocardium via Gap Junction Modification," Tissue Engineering Part: A, vol. 15, no. 10, pp. 3099-3108, 2009.

[46] D.-H. Kim, E. A. Lipke, P. Kim et al., "Nanoscale cues regulate the structure and function of macroscopic cardiac tissue constructs," Proceedings of the National Acadamy of Sciences of the United States of America, vol. 107, no. 2, pp. 565-570, 2010.

[47] V. Mironov, R. P. Visconti, V. Kasyanov, G. Forgacs, C. J. Drake, and R. R. Markwald, "Organ printing: tissue spheroids as building blocks," Biomaterials, vol. 30, no. 12, pp. 2164-2174, 2009.

[48] R. Noguchi, K. Nakayama, M. Itoh et al., "Development of a three-dimensional pre-vascularized scaffold-free contractile cardiac patch for treating heart disease," The Journal of Heart and Lung Transplantation, vol. 35, no. 1, pp. 137-145, 2016.

[49] A. Elamparithi, A. M. Punnoose, and S. Kuruvilla, "Electrospun type 1 collagen matrices preserving native ultrastructure using benign binary solvent for cardiac tissue engineering," Artificial Cells, Nanomedicine and Biotechnology, vol. 44, no. 5, pp. 13181325, 2016.

[50] E. Cambria, F. S. Pasqualini, P. Wolint et al., "Translational cardiac stem cell therapy: advancing from first-generation to next-generation cell types," npj Regenerative Medicine, vol. 2, no. $1,2017$.

[51] K. Rajala, M. Pekkanen-Mattila, and K. Aalto-Setälä, "Cardiac differentiation of pluripotent stem cells," Stem Cells International, vol. 2011, Article ID 383709, p. 12, 2011.

[52] C. L. Mummery, J. Zhang, E. S. Ng, D. A. Elliott, A. G. Elefanty, and T. J. Kamp, "Differentiation of human embryonic stem cells and induced pluripotent stem cells to cardiomyocytes: a methods overview," Circulation Research, vol. 111, no. 3, pp. 344358, 2012.

[53] X. Lian, C. Hsiao, G. Wilson et al., "Robust cardiomyocyte differentiation from human pluripotent stem cells via temporal modulation of canonical Wnt signaling," Proceedings of the National Acadamy of Sciences of the United States of America, vol. 109, no. 27, pp. E1848-E1857, 2012.

[54] L. W. van Laake, R. Passier, J. Monshouwer-Kloots et al., "Human embryonic stem cell-derived cardiomyocytes survive and mature in the mouse heart and transiently improve function after myocardial infarction," Stem Cell Research, vol. 1, no. 1, pp. 9-24, 2007.

[55] L. W. Van Laake, R. Passier, P. A. Doevendans, and C. L. Mummery, "Human embryonic stem cell-derived cardiomyocytes and cardiac repair in rodents," Circulation Research, vol. 102, no. 9, pp. 1008-1010, 2008.

[56] S. Der Sarkissian, T. Lévesque, and N. Noiseux, "Optimizing stem cells for cardiac repair: Current status and new frontiers in regenerative cardiology," World Journal of Stem Cells, vol. 9, no. 1, p. 9, 2017.

[57] F. B. Bedada, M. Wheelwright, and J. M. Metzger, "Maturation status of sarcomere structure and function in human iPSCderived cardiac myocytes," Biochimica et Biophysica Acta (BBA) - Molecular Cell Research, vol. 1863, no. 7, pp. 1829-1838, 2016.

[58] F. X. Galdos, Y. Guo, S. L. Paige, N. J. Vandusen, S. M. Wu, and W. T. Pu, "Cardiac Regeneration: Lessons from Development," Circulation Research, vol. 120, no. 6, pp. 941-959, 2017.

[59] J. Chong, X. Yang, C. Don, E. Minami, B. Van Biber, and C. Murry, "PW281 Human Pluripotent Stem Cell Derived 
Cardiomyocytes Regenerate Infarcted Hearts Of Non-Human Primates," Global Heart, vol. 9, no. 1, pp. e315-e316, 2014.

[60] R. Duelen and M. Sampaolesi, "Stem Cell Technology in Cardiac Regeneration: A Pluripotent Stem Cell Promise," EBioMedicine, vol. 16, pp. 30-40, 2017.

[61] O. Bergmann, S. Zdunek, A. Felker et al., "Dynamics of cell generation and turnover in the human heart," Cell, vol. 161, no. 7, pp. 1566-1575, 2015.

[62] S. Walsh, A. Pontén, B. K. Fleischmann, and S. Jovinge, "Cardiomyocyte cell cycle control and growth estimation in vivoAn analysis based on cardiomyocyte nuclei," Cardiovascular Research, vol. 86, no. 3, pp. 365-373, 2010.

[63] I. Banerjee, J. W. Fuseler, R. L. Price, T. K. Borg, and T. A. Baudino, "Determination of cell types and numbers during cardiac development in the neonatal and adult rat and mouse," American Journal of Physiology-Heart and Circulatory Physiology, vol. 293, no. 3, pp. H1883-H1891, 2007.

[64] C. A. Souders, T. K. Borg, I. Banerjee, and T. A. Baudino, "Pressure overload induces early morphological changes in the heart," The American Journal of Pathology, vol. 181, no. 4, pp. 1226-1235, 2012.

[65] A. R. Pinto, A. Ilinykh, M. J. Ivey et al., "Revisiting cardiac cellular composition," Circulation Research, vol. 118, no. 3, pp. 400-409, 2016.

[66] P. Anversa, G. Olivetti, M. Melissari, and A. V. Loud, "Stereological measurement of cellular and subcellular hypertrophy and hyperplasia in the papillary muscle of adult rat," Journal of Molecular and Cellular Cardiology, vol. 12, no. 8, pp. 781-795, 1980.

[67] J. Dämmrich and U. Pfeifer, "Cardiac hypertrophy in rats after supravalvular aortic constriction - II. Inhibition of cellular autophagy in hypertrophying cardiomyocytes," Virchows Archiv B Cell Pathology Including Molecular Pathology, vol. 43, no. 1, pp. 287-307, 1983.

[68] T. Mattfeldt, K.-L. Krämer, R. Zeitz, and G. Mall, "Stereology of myocardial hypertrophy induced by physical exercise," Virchows Archiv A Pathological Anatomy and Histopathology, vol. 409, no. 4, pp. 473-484, 1986.

[69] N. L. Tulloch, V. Muskheli, M. V. Razumova et al., "Growth of engineered human myocardium with mechanical loading and vascular coculture," Circulation Research, vol. 109, no. 1, pp. 4759, 2011.

[70] A. Lesman, M. Habib, O. Caspi et al., "Transplantation of a tissue-engineered human vascularized cardiac muscle," Tissue Engineering Part: A, vol. 16, no. 1, pp. 115-125, 2010.

[71] M. Radisic, A. Marsano, R. Maidhof, Y. Wang, and G. VunjakNovakovic, "Cardiac tissue engineering using perfusion bioreactor systems," Nature Protocols, vol. 3, no. 4, pp. 719-738, 2008.

[72] H. Naito, I. Melnychenko, M. Didié et al., "Optimizing engineered heart tissue for therapeutic applications as surrogate heart muscle," Circulation, vol. 114, no. 1, pp. I72-I78, 2006.

[73] O. Caspi, A. Lesman, Y. Basevitch et al., "Tissue engineering of vascularized cardiac muscle from human embryonic stem cells," Circulation Research, vol. 100, no. 2, pp. 263-272, 2007.

[74] S. C. Hung, R. R. Pochampally, S. C. Chen, S. C. Hsu, and D. J. Prockop, "Angiogenic effects of human multipotent stromal cell conditioned medium activate the PI3K-Akt pathway in hypoxic endothelial cells to inhibit apoptosis, increase survival, and stimulate angiogenesis," Stem Cells, vol. 25, no. 9, pp. 2363-2370, 2007.
[75] R. K. Jain, P. Au, J. Tam, D. G. Duda, and D. Fukumura, "Engineering vascularized tissue," Nature Biotechnology, vol. 23, no. 7, pp. 821-823, 2005.

[76] S. Levenberg, J. Rouwkema, M. Macdonald et al., "Engineering vascularized skeletal muscle tissue," Nature Biotechnology, vol. 23, no. 7, pp. 879-884, 2005.

[77] L. L. Y. Chiu and M. Radisic, "Scaffolds with covalently immobilized VEGF and Angiopoietin-1 for vascularization of engineered tissues," Biomaterials, vol. 31, no. 2, pp. 226-241, 2010.

[78] Y. Assal, M. Mie, and E. Kobatake, “The promotion of angiogenesis by growth factors integrated with ECM proteins through coiled-coil structures," Biomaterials, vol. 34, no. 13, pp. 33153323, 2013.

[79] M. K. Schesny, M. Monaghan, A. H. Bindermann et al., "Preserved bioactivity and tunable release of a SDF1-GPVI bi-specific protein using photo-crosslinked PEGda hydrogels," Biomaterials, vol. 35, no. 25, pp. 7180-7187, 2014.

[80] E. A. Phelps and A. J. García, "Engineering more than a cell: vascularization strategies in tissue engineering," Current Opinion in Biotechnology, vol. 21, no. 5, pp. 704-709, 2010.

[81] J. E. Leslie-Barbick, C. Shen, C. Chen, and J. L. West, "Micronscale spatially patterned, covalently immobilized vascular endothelial growth factor on hydrogels accelerates endothelial tubulogenesis and increases cellular angiogenic responses," Tissue Engineering Part: A, vol. 17, no. 1-2, pp. 221-229, 2011.

[82] W. Mulyasasmita, L. Cai, R. E. Dewi et al., "Avidity-controlled hydrogels for injectable co-delivery of induced pluripotent stem cell-derived endothelial cells and growth factors," Journal of Controlled Release, vol. 191, pp. 71-81, 2014.

[83] J. W. Macarthur, J. E. Cohen, J. R. Mcgarvey et al., "Preclinical evaluation of the engineered stem cell chemokine stromal cellderived factor $1 \alpha$ analog in a translational ovine myocardial infarction model," Circulation Research, vol. 114, no. 4, pp. 650 659, 2014.

[84] K. M. Chrobak, D. R. Potter, and J. Tien, "Formation of perfused, functional microvascular tubes in vitro," Microvascular Research, vol. 71, no. 3, pp. 185-196, 2006.

[85] J. H. Park, B. G. Chung, W. G. Lee et al., "Microporous cell-laden hydrogels for engineered tissue constructs," Biotechnology and Bioengineering, vol. 106, no. 1, pp. 138-148, 2010.

[86] A. P. Golden and J. Tien, "Fabrication of microfluidic hydrogels using molded gelatin as a sacrificial element," Lab on a Chip , vol. 7, no. 6, pp. 720-725, 2007.

[87] J. S. Miller, K. R. Stevens, M. T. Yang et al., "Rapid casting of patterned vascular networks for perfusable engineered threedimensional tissues," Nature Materials, vol. 11, no. 9, pp. 768774, 2012.

[88] J. C. Culver, J. C. Hoffmann, R. A. Poché, J. H. Slater, J. L. West, and M. E. Dickinson, "Three-dimensional biomimetic patterning in hydrogels to guide cellular organization," Advanced Materials, vol. 24, no. 17, pp. 2344-2348, 2012.

[89] K. C. Hribar, K. Meggs, J. Liu, W. Zhu, X. Qu, and S. Chen, "Three-dimensional direct cell patterning in collagen hydrogels with near-infrared femtosecond laser," Scientific Reports, vol. 5, Article ID 17203, 2015.

[90] K. A. Garvin, D. C. Hocking, and D. Dalecki, "Controlling the spatial organization of cells and extracellular matrix proteins in engineered tissues using ultrasound standing wave fields," Ultrasound in Medicine \& Biology, vol. 36, no. 11, pp. 1919-1932, 2010. 
[91] K. A. Garvin, D. Dalecki, and D. C. Hocking, "Vascularization of three-dimensional collagen hydrogels using ultrasound standing wave fields.," Ultrasound in medicine \& biology, vol. 37, no. 11, pp. 1853-1864, 2011.

[92] K. A. Garvin, D. Dalecki, M. Yousefhussien, M. Helguera, and D. C. Hocking, "Spatial patterning of endothelial cells and vascular network formation using ultrasound standing wave fields," The Journal of the Acoustical Society of America, vol. 134, no. 2, pp. 1483-1490, 2013.

[93] E. S. Comeau, D. C. Hocking, and D. Dalecki, "Ultrasound patterning technologies for studying vascular morphogenesis in 3D," Journal of Cell Science, vol. 130, no. 1, pp. 232-242, 2017.

[94] W.-H. Zimmermann, I. Melnychenko, G. Wasmeier et al., "Engineered heart tissue grafts improve systolic and diastolic function in infarcted rat hearts," Nature Medicine, vol. 12, no. 4, pp. 452-458, 2006.

[95] W.-H. Zimmermann, M. Didié, G. H. Wasmeier et al., "Cardiac grafting of engineered heart tissue in syngenic rats," Circulation, vol. 106, Suppl 1, no. 13, pp. I151-I157, 2002.

[96] T. Shimizu, H. Sekine, J. Yang et al., "Polysurgery of cell sheet grafts overcomes diffusion limits to produce thick, vascularized myocardial tissues," The FASEB Journal, vol. 20, no. 6, pp. 708710, 2006

[97] R. Tee, W. A. Morrison, G. J. Dusting et al., "Transplantation of engineered cardiac muscle flaps in syngeneic rats," Tissue Engineering Part: A, vol. 18, no. 19-20, pp. 1992-1999, 2012.

[98] H. Sekine, T. Shimizu, K. Sakaguchi et al., "In vitro fabrication of functional three-dimensional tissues with perfusable blood vessels," Nature Communications, vol. 4, article no. 1399, 2013.

[99] L. L. Y. Chiu, M. Montgomery, Y. Liang, H. Liu, and M. Radisic, "Perfusable branching microvessel bed for vascularization of engineered tissues," Proceedings of the National Acadamy of Sciences of the United States of America, vol. 109, no. 50, pp. E3414-E3423, 2012.

[100] K. Sakaguchi, T. Shimizu, S. Horaguchi et al., "In vitro engineering of vascularized tissue surrogates," Scientific Reports, vol. 3, article no. 1316, 2013.

[101] B. Gao, Q. Yang, X. Zhao, G. Jin, Y. Ma, and F. Xu, “4D Bioprinting for Biomedical Applications," Trends in Biotechnology, vol. 34, no. 9, pp. 746-756, 2016.

[102] Z. X. Khoo, J. E. Teoh, Y. Liu et al., "3D printing of smart materials: A review on recent progresses in 4D printing," Virtual and Physical Prototyping, vol. 10, no. 3, pp. 103-122, 2015.

[103] J. An, C. K. Chua, and V. Mironov, "A Perspective on 4D Bioprinting," International Journal of Bioprinting, vol. 2, no. 0, 2016.

[104] A. Kirillova, R. Maxson, G. Stoychev, C. T. Gomillion, and L. Ionov, "4D Biofabrication Using Shape-Morphing Hydrogels," Advanced Materials, vol. 29, no. 46, Article ID 1703443, 2017.

[105] I. Apsite, G. Stoychev, W. Zhang, D. Jehnichen, J. Xie, and L. Ionov, "Porous Stimuli-Responsive Self-Folding Electrospun Mats for 4D Biofabrication," Biomacromolecules, vol. 18, no. 10, pp. 3178-3184, 2017.

[106] Y. Wang, C. K. Adokoh, and R. Narain, "Recent development and biomedical applications of self-healing hydrogels," Expert Opinion on Drug Delivery, vol. 15, no. 1, pp. 77-91, 2017.

[107] K. Y. Ye, K. E. Sullivan, and L. D. Black, "Encapsulation of cardiomyocytes in a fibrin hydrogel for cardiac tissue engineering," Journal of Visualized Experiments, no. 55, Article ID e3251, 2011.

[108] H. Kaji, Y. Takii, M. Nishizawa, and T. Matsue, "Pharmacological characterization of micropatterned cardiac myocytes," Biomaterials, vol. 24, no. 23, pp. 4239-4244, 2003.
[109] V. Serpooshan, P. Chen, H. Wu et al., "Bioacoustic-enabled patterning of human iPSC-derived cardiomyocytes into 3D cardiac tissue," Biomaterials, vol. 131, pp. 47-57, 2017.

[110] H. C. Ott, T. S. Matthiesen, S.-K. Goh et al., "Perfusiondecellularized matrix: using nature's platform to engineer a bioartificial heart," Nature Medicine, vol. 14, no. 2, pp. 213-221, 2008.

[111] T. R. Olsen, B. Mattix, M. Casco et al., "Manipulation of cellular spheroid composition and the effects on vascular tissue fusion," Acta Biomaterialia, vol. 13, pp. 188-198, 2015.

[112] M. Itoh, K. Nakayama, R. Noguchi et al., "Scaffold-free tubular tissues created by a bio-3D printer undergo remodeling and endothelialization when implanted in rat aortae," PLOS ONE, vol. 10, no. 9, e0136681 pages, 2015.

[113] M. Tiburcy, J. E. Hudson, P. Balfanz et al., "Defined engineered human myocardium with advanced maturation for applications in heart failure modeling and repair," Circulation, vol.135, no. 19, pp. 1832-1847, 2017. 


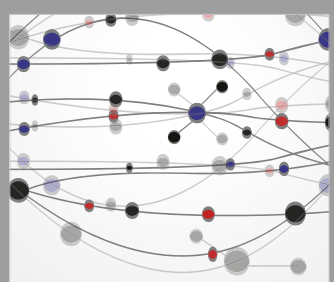

The Scientific World Journal
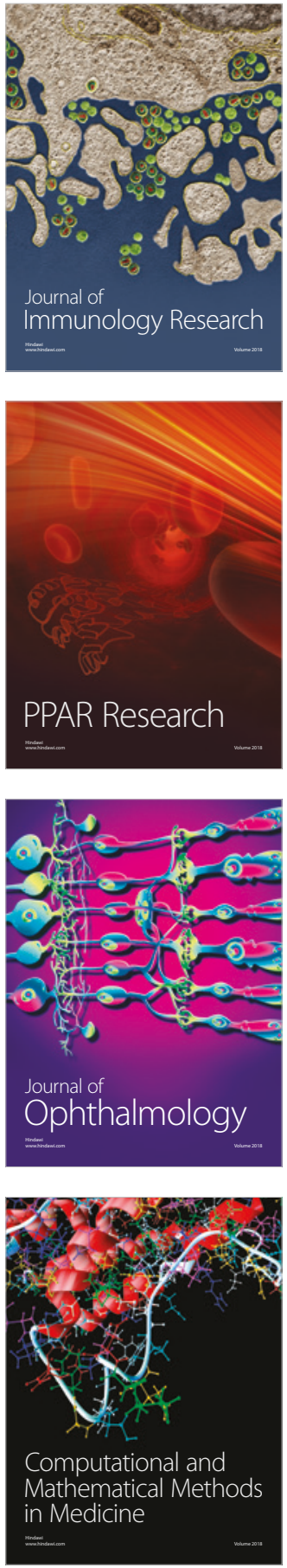

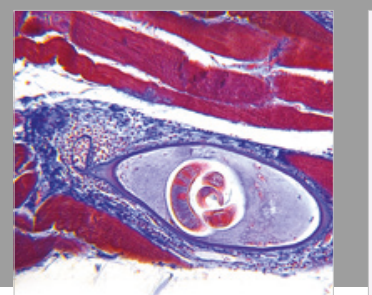

Gastroenterology Research and Practice

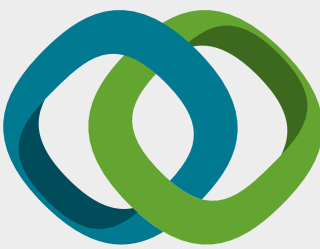

\section{Hindawi}

Submit your manuscripts at

www.hindawi.com
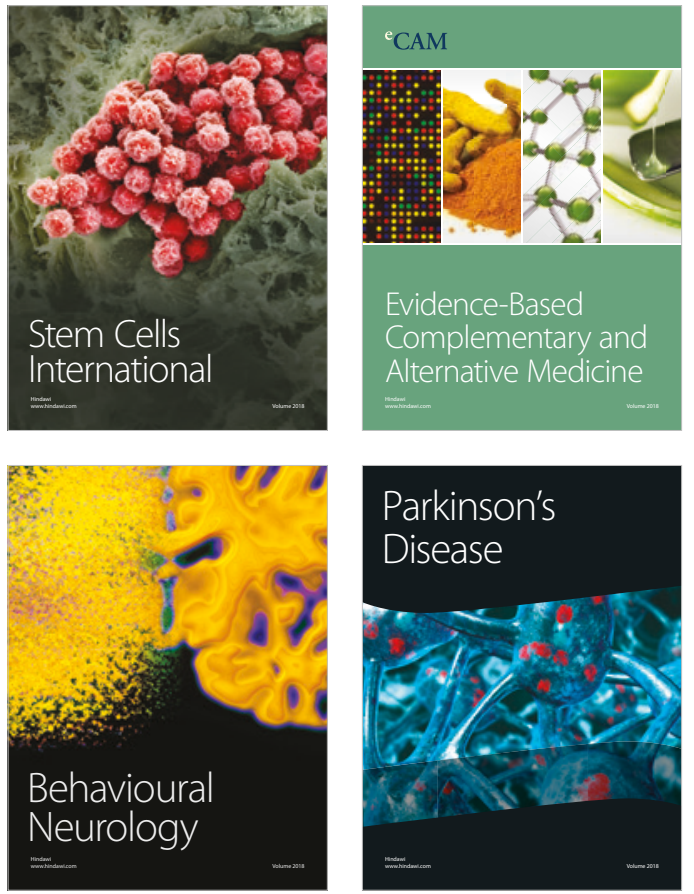

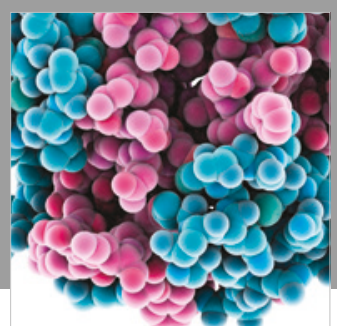

ournal of

Diabetes Research

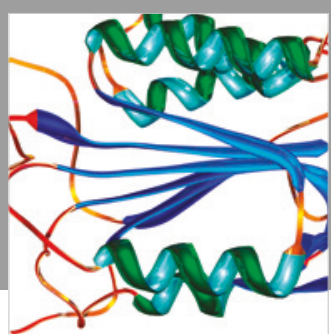

Disease Markers
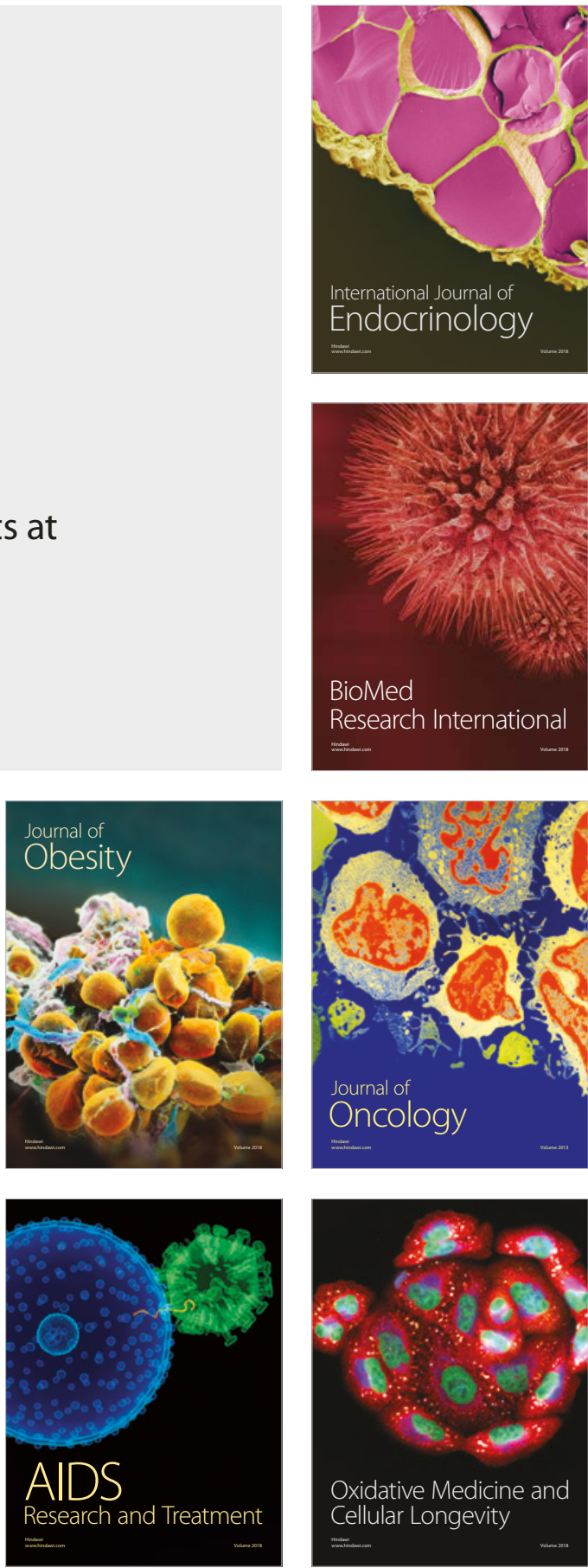